75

ournal of

Mechanics of

Materials and Structures

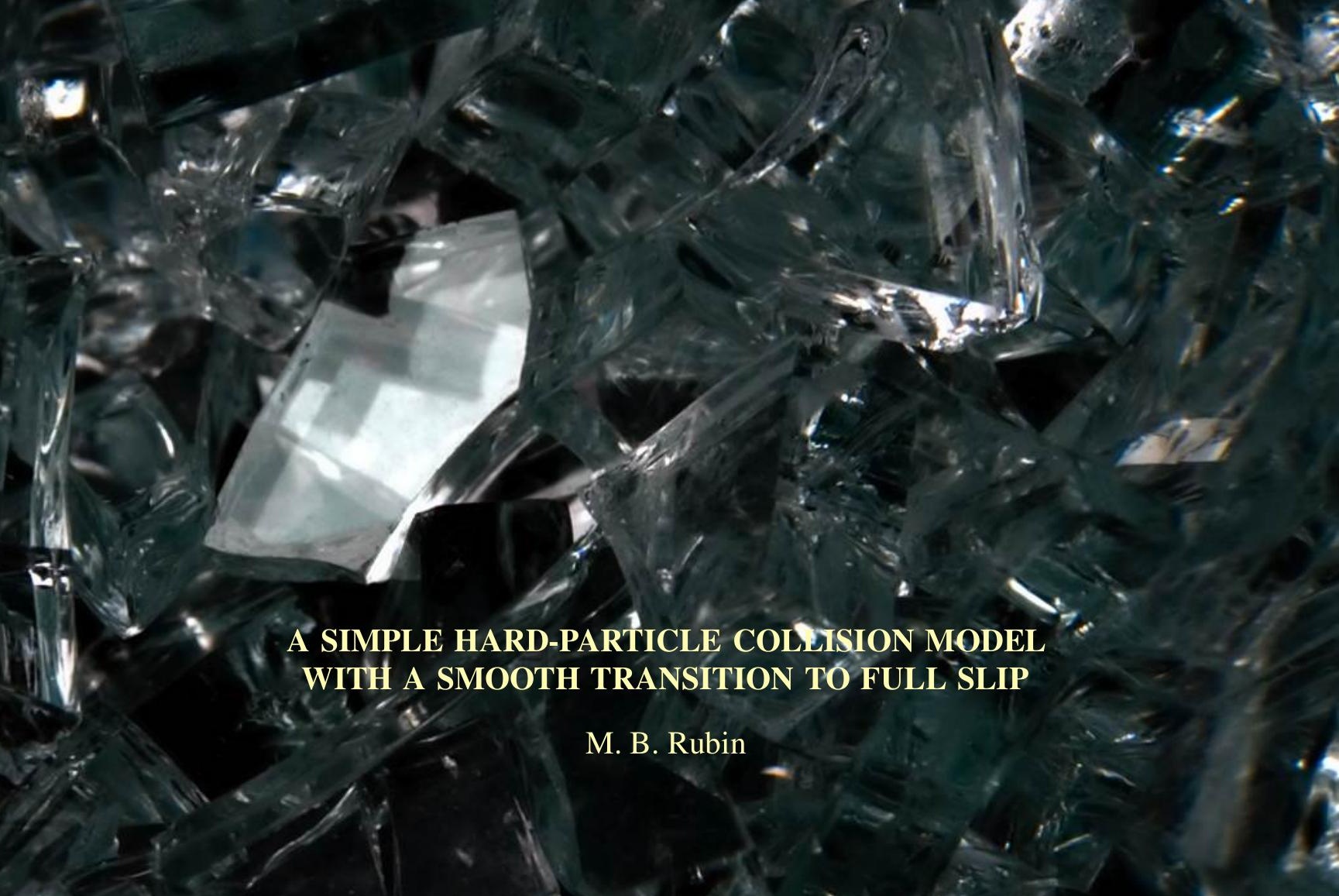

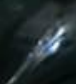

(a) 8<smiles>C1=C[As]C1</smiles>

11 , 2 ,

Volume 11 , No. $2, \ldots$

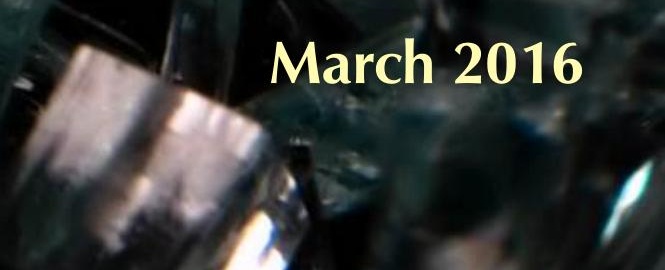

. 


\title{
A SIMPLE HARD-PARTICLE COLLISION MODEL WITH A SMOOTH TRANSITION TO FULL SLIP
}

\author{
M. B. RUBIN
}

\begin{abstract}
Hard-particle models assume that the collision between rigid bodies is instantaneous and they propose algebraic equations for the jumps in the motions of the rigid bodies based on an assumed functional form for the impulsive force acting during the collision process. These hard-particle models have the advantage that they are significantly less computationally expensive than soft-particle models. Moreover, they are sufficiently accurate for some applications and can also be used for preliminary analysis of complicated processes with large numbers of particle interactions. Here, a simple three parameter hard-particle collision model is developed which predicts a smooth transition from normal collisions with no slip to oblique collisions with full slip. This model automatically satisfies a number of physical restrictions on the impulsive force. An additional physical restriction on nonnegative energy dissipation places nontrivial restrictions on the model parameters. Examples show the importance of improved modeling of the transition region from normal to oblique collisions with full slip.
\end{abstract}

\section{Introduction}

The mechanics of collision of two bodies is complicated by dynamic wave propagation, stick-slip frictional contact conditions and plastic dissipation [Goldsmith 1960]. The Distinct Element Method (DEM) is currently being used to model a broad range of physical phenomena like: granular flow [Walton 2004; Di Renzo and Di Maio 2004; Ketterhagen et al. 2005], particle fluids [Lu et al. 2005; Prasanth et al. 2012], studies of agglomeration [Kosinski and Hoffmann 2010; 2011], powder technology [Liu et al. 2011] and shock waves in jointed geological media [Morris et al. 2004]. Most often the DEM methods model the interaction of collections of rigid bodies with various shapes. The equations of motion of the rigid bodies are integrated using constitutive equations for the interactions between bodies that are in contact with each other. Soft-particle contact models use information about the virtual penetration regions of the bodies to determine the normal and shearing components of the contact force. The constitutive equations for the contact force attempt to model details of elastic and inelastic effects that are relevant for the specific application. In addition to the extensive computational effort to determine the contact regions, it is necessary to use small time steps to accurately integrate the equations of motion over the short interaction times associated with abrupt collision events.

The computational effort in DEM calculations to identify and analyze the virtual penetration regions can be significantly reduced when the bodies have simple shapes like spheres. Additional reduction in computational effort can be obtained by simplifying the contact events as instantaneous collisions. This simplification uses hard-particle models which assume that the contact region is small enough that only

Keywords: coefficient of restitution, collision, friction, hard-particle, rigid bodies, spheres. 
a single force acts at the point of contact between the bodies. Also, it is assumed that the collision events are separated in time so that at any instant only a single contact process operates between two bodies. Details of the contact event are modeled by proposing constitutive equations for the impulsive force that acts between the bodies at the point of contact. Then, the equations of motion of the rigid bodies are integrated to obtain algebraic equations that predict the motion of the bodies just after the collision in terms of the motion of the bodies just before the collision. Hard-particle models have the advantage that they are significantly less computationally expensive than soft-particle models. Moreover, they are sufficiently accurate for some applications and can also be used for preliminary analysis of complicated processes with large numbers of particle interactions.

A review of the numerical simulation of inelastic, frictional particle-particle interactions can be found in [Walton 1993]. More recent reviews discuss: discrete particle modeling of fluidized beds [Deen et al. 2007] the extension of normal force models [Kruggel-Emden et al. 2007]; the simulation of particulate systems [Zhu et al. 2007]; and particle collision modeling [Meyer and Deglon 2011]. Ketterhagen et al. [2005] studied flows with a range of solid fractions to examine the limits of hard-particle collision models, which are typically valid for dilute systems. Also, it is noted that Stevens and Hrenya [2005] have presented a comparison of predictions of soft-sphere models and measurements of normal impacts.

The algebraic equations that describe the jumps in the motion of two colliding rigid bodies have been discussed in [Rubin 1998; Chatterjee and Ruina 1998]. These equations are based on an energetic coefficient of restitution $\eta$ introduced by Ivanov [1992]. More specifically, the work in [Rubin 1998] discusses physical restrictions on constitutive equations for the impulsive force acting between the two rigid bodies during hard-particle collision events.

Walton [1993, sec. 25.5.1] discusses a hard-particle model for the collision of spheres that is based on the elastic, frictional solutions of the dynamic equations of elasticity described in [Maw et al. 1976; 1981]. This model depends on three parameters: the usual coefficient of restitution $e$, which connects jumps in the normal component of the relative velocity of the contact points of the bodies; a coefficient of rotational restitution $\beta$, which connects jumps in the tangential component of the relative velocity of the contact points of the bodies; and the constant shear coefficient $\mu_{s}$ which controls the ratio of the shear component of the impulsive force to its normal component in a full slip region. More specifically, the model is based on two straight lines in the space of auxiliary parameters $\left\{\psi_{1}, \psi_{2}\right\}$, which are measures of the obliquity of the collision event at the point of contact, just before and just after collision, respectively. One line characterizes oblique collisions with full slip and the other line characterizes partial slip during the transition from normal to oblique collisions with full slip. In particular, the model has a sharp transition at the intersection of these two lines.

The objective of the present paper is to analyze the model proposed in [Walton 1993] from the perspective of the physical restrictions discussed in [Rubin 1998]. This analysis reveals that the transition region from zero slip to full slip is large with respect to the angle of obliquity. Consequently, it is important to improve the modeling of this transition region from normal to oblique collisions with full slip. Moreover, the present work suggests that the rotational coefficient of restitution be replaced by a shear coefficient $\mu$, which characterizes the ratio of the shear component of the impulsive force to its normal component. This shear coefficient increases from zero for normal collisions to a maximum constant value $\mu_{s}$ for oblique collisions with full slip. Specifically, a three parameter model is proposed with a smooth transition from no slip to full slip. All three parameters can be determined directly from experimental data or from 

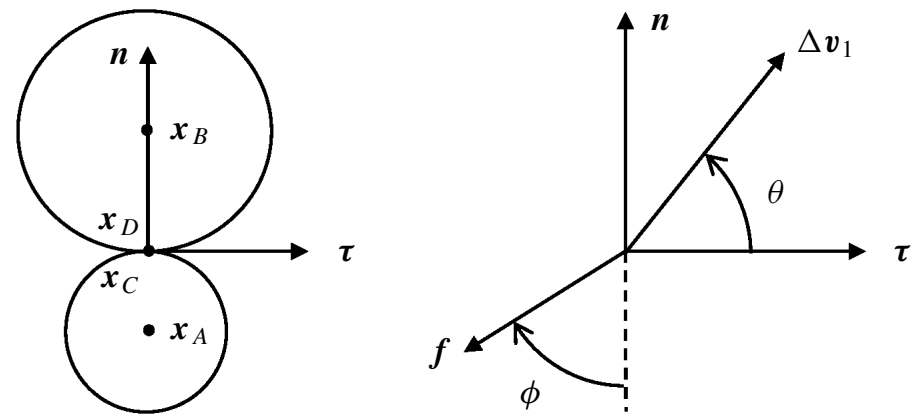

Figure 1. Sketch of the spheres, the unit outward normal vector $\boldsymbol{n}$ to sphere $(A)$, the unit tangent vector $\tau$ and the directions of $\Delta \boldsymbol{v}_{1}$ and $f$.

results of simulations using more complicated soft-particle models. In addition, a restriction is imposed on the model parameters which ensures non-negative energy dissipation in the collision event.

An outline of this paper is as follows. Section 2 reviews the basic equations of the formulation in [Rubin 1998] and specializes these equations to collision of two spheres. In particular, this section reviews physical restrictions that are placed on constitutive equations for the impulsive force acting during the collision event. Section 3 proposes a constitutive equation for the impulsive force that exhibits a smooth transition from normal to oblique collisions. Section 4 reviews the hard-particle model of Walton [1993] and Section 5 compares the smooth transition model with that of Walton. Section 6 considers the example of a sphere that bounces off a fixed horizontal plane and Section 7 presents conclusions.

\section{Basic equations}

In this section the general formulation presented in [Rubin 1998] is specialized to the case of collision of two rigid spheres. For simplicity, a number of equations are summarized without further reference to the work in [Rubin 1998]. Specifically, consider two spherical rigid bodies with masses $\left\{m_{A}, m_{B}\right\}$, radii $\left\{R_{A}, R_{B}\right\}$ and moments of inertia $\left\{I_{A}, I_{B}\right\}$ about their centers of mass, which have positions $\left\{\boldsymbol{x}_{A}, \boldsymbol{x}_{B}\right\}$ relative to a fixed origin (see Figure 1). Just before collision, the centers of mass have velocities $\left\{\boldsymbol{v}_{A 1}, \boldsymbol{v}_{B 1}\right\}$ and the bodies have angular velocities $\left\{\omega_{A 1}, \omega_{B 1}\right\}$. Just after collision, the centers of mass have velocities $\left\{\boldsymbol{v}_{A 2}, \boldsymbol{v}_{B 2}\right\}$ and the bodies have angular velocities $\left\{\boldsymbol{\omega}_{A 2}, \boldsymbol{\omega}_{B 2}\right\}$.

The collision event is considered to be instantaneous with only a point force acting between the bodies. Specifically, let $\hat{\boldsymbol{F}}$ denote the impulsive force applied by body $B$ on body $A$ at the common contact point which is denoted as $\boldsymbol{x}_{C}$ for the material point attached to body $A$ and by $\boldsymbol{x}_{D}$ for the material point attached to body $B$. Also, let $\boldsymbol{n}$ be the unit outward normal to the body $A$ at the impact point $\boldsymbol{x}_{C}=\boldsymbol{x}_{D}$, such that

$$
\boldsymbol{n}=\frac{\boldsymbol{x}_{B / A}}{\left|\boldsymbol{x}_{B / A}\right|}, \quad \boldsymbol{n} \cdot \boldsymbol{n}=1, \quad \boldsymbol{x}_{C / A}=R_{A} \boldsymbol{n}, \quad \boldsymbol{x}_{D / B}=-R_{B} \boldsymbol{n},
$$

where for simplicity use has been made of the notation

$$
x_{B / A}=x_{B}-x_{A} .
$$


The impulsive forms of the balances of linear and angular momentum (about the centers of mass) can be solved to deduce that

$$
\begin{array}{rlrl}
\boldsymbol{v}_{A 2} & =\boldsymbol{v}_{A 1}+\frac{1}{m_{A}} \hat{\boldsymbol{F}}, & \boldsymbol{v}_{B 2} & =\boldsymbol{v}_{B 1}-\frac{1}{m_{B}} \hat{\boldsymbol{F}}, \\
\boldsymbol{\omega}_{A 2} & =\boldsymbol{\omega}_{A 1}+\frac{R_{A}}{I_{A}} \boldsymbol{n} \times \hat{\boldsymbol{F}}, & \boldsymbol{\omega}_{B 2}=\boldsymbol{\omega}_{B 1}+\frac{R_{B}}{I_{B}} \boldsymbol{n} \times \hat{\boldsymbol{F}} .
\end{array}
$$

Moreover, the velocities $\left\{\boldsymbol{v}_{C 1}, \boldsymbol{v}_{D 1}\right\}$ of the contact material points just before the collision and the velocities $\left\{\boldsymbol{v}_{C 2}, \boldsymbol{v}_{D 2}\right\}$ just after the collision are given by

$$
\begin{array}{ll}
\boldsymbol{v}_{C 1}=\boldsymbol{v}_{A 1}+R_{A} \boldsymbol{\omega}_{A 1} \times \boldsymbol{n}, & \boldsymbol{v}_{D 1}=\boldsymbol{v}_{B 1}-R_{B} \boldsymbol{\omega}_{B 1} \times \boldsymbol{n}, \\
\boldsymbol{v}_{C 2}=\boldsymbol{v}_{A 2}+R_{A} \boldsymbol{\omega}_{A 2} \times \boldsymbol{n}, & \boldsymbol{v}_{D 2}=\boldsymbol{v}_{B 2}-R_{B} \boldsymbol{\omega}_{B 2} \times \boldsymbol{n} .
\end{array}
$$

Next, it is convenient to define the velocity differences of the contact points $\left\{\Delta \boldsymbol{v}_{1}, \Delta \boldsymbol{v}_{2}\right\}$ just before and just after the collision by the expressions

$$
\Delta \boldsymbol{v}_{1}=\boldsymbol{v}_{C 1}-\boldsymbol{v}_{D 1}, \quad \Delta \boldsymbol{v}_{2}=\boldsymbol{v}_{C 2}-\boldsymbol{v}_{D 2},
$$

and to recall that

$$
\Delta \boldsymbol{v}_{2}=\Delta \boldsymbol{v}_{1}+\frac{1}{m} \boldsymbol{A} \hat{\boldsymbol{F}}, \quad \boldsymbol{A}=\left(\frac{1+I_{m}}{I_{m}}\right) \boldsymbol{I}-\left(\frac{1}{I_{m}}\right) \boldsymbol{n} \otimes \boldsymbol{n},
$$

where $\boldsymbol{A}$ is a second order tensor and the effective mass $m$ for linear momentum and the parameter $I_{m}$ associated with angular momentum are defined by

$$
m=\frac{m_{A} m_{B}}{m_{A}+m_{B}}, \quad I_{m}=\frac{I_{A} I_{B}}{m\left(I_{A} R_{B}^{2}+I_{B} R_{A}^{2}\right)} .
$$

Also, $\boldsymbol{a} \otimes \boldsymbol{b}$ denotes the tensor product between two vectors $\{\boldsymbol{a}, \boldsymbol{b}\}$. Here, the spherical bodies are assumed to have spherically symmetric mass distributions but are allowed to have inhomogeneous mass distributions radially.

The incident slip velocity $\Delta \boldsymbol{v}_{1}$ and the unit normal $\boldsymbol{n}$ form a plane which contains the slip direction $\tau$, such that

$$
\Delta \boldsymbol{v}_{1}=\frac{\left|\Delta \boldsymbol{v}_{1}\right|}{\sqrt{1+\psi_{1}^{2}}}\left(\boldsymbol{n}+\psi_{1} \boldsymbol{\tau}\right), \quad \psi_{1}=\tan \theta \geq 0, \quad \boldsymbol{\tau}=\frac{\Delta \boldsymbol{v}_{1}-\left(\Delta \boldsymbol{v}_{1} \cdot \boldsymbol{n}\right) \boldsymbol{n}}{\left|\Delta \boldsymbol{v}_{1}-\left(\Delta \boldsymbol{v}_{1} \cdot \boldsymbol{n}\right) \boldsymbol{n}\right|}
$$

where $\theta$ is the incident angle of obliquity (see Figure 1). It follows that since

$$
\Delta \boldsymbol{v}_{1} \cdot \boldsymbol{n}>0
$$

sphere $A$ collides with sphere $B$.

Denoting $\left\{T_{1}, T_{2}\right\}$ as the kinetic energy of the two-particle system just before and just after the collision

$$
\begin{aligned}
& T_{1}=\frac{1}{2} m_{A} \boldsymbol{v}_{A 1} \cdot \boldsymbol{v}_{A 1}+\frac{1}{2} I_{A} \boldsymbol{\omega}_{A 1} \cdot \boldsymbol{\omega}_{A 1}+\frac{1}{2} m_{B} \boldsymbol{v}_{B 1} \cdot \boldsymbol{v}_{B 1}+\frac{1}{2} I_{B} \boldsymbol{\omega}_{B 1} \cdot \boldsymbol{\omega}_{B 1}, \\
& T_{2}=\frac{1}{2} m_{A} \boldsymbol{v}_{A 2} \cdot \boldsymbol{v}_{A 2}+\frac{1}{2} I_{A} \boldsymbol{\omega}_{A 2} \cdot \boldsymbol{\omega}_{A 2}+\frac{1}{2} m_{B} \boldsymbol{v}_{B 2} \cdot \boldsymbol{v}_{B 2}+\frac{1}{2} I_{B} \boldsymbol{\omega}_{B 2} \cdot \boldsymbol{\omega}_{B 2},
\end{aligned}
$$


it can be shown that

$$
T_{1}-T_{2}=-\hat{\boldsymbol{F}} \cdot \Delta \boldsymbol{v}_{1}-\frac{1}{2 m} \boldsymbol{A} \cdot(\hat{\boldsymbol{F}} \otimes \hat{\boldsymbol{F}}),
$$

where $\boldsymbol{A} \cdot(\boldsymbol{a} \otimes \boldsymbol{b})=\operatorname{tr}\left[\boldsymbol{A}(\boldsymbol{a} \otimes \boldsymbol{b})^{T}\right]$ is the inner product between the two second order tensors $\{\boldsymbol{A}, \boldsymbol{a} \otimes \boldsymbol{b}\}$.

In [Rubin 1998] $\hat{\boldsymbol{F}}$ was expressed in the form

$$
\hat{\boldsymbol{F}}=f \boldsymbol{f}, \quad \boldsymbol{f} \cdot \boldsymbol{f}=1, \quad f=m(1+\eta)\left[\frac{-\boldsymbol{f} \cdot \Delta \boldsymbol{v}_{1}}{\boldsymbol{A} \cdot(\boldsymbol{f} \otimes \boldsymbol{f})}\right]>0,
$$

which can be used to show that (2-11) reduces to

$$
T_{1}-T_{2}=\frac{1}{2} m\left|\Delta \boldsymbol{v}_{1}\right|^{2} \Delta T, \quad \Delta T=\frac{1}{2} m\left(1-\eta^{2}\right)\left[\frac{\left(\boldsymbol{f} \cdot \Delta \boldsymbol{v}_{1} /\left|\Delta \boldsymbol{v}_{1}\right|\right)^{2}}{\boldsymbol{A} \cdot(\boldsymbol{f} \otimes \boldsymbol{f})}\right] .
$$

In particular, the work in [Rubin 1998] suggests that the constitutive equation for the impulsive force must satisfy the following four physical restrictions.

(P1) The impulse is not orthogonal to the relative velocity of the contact points just before the collision

$$
\hat{\boldsymbol{F}} \cdot \Delta \boldsymbol{v}_{1} \neq 0
$$

(P2) The impulse has a component that resists penetration and aids separation of the two bodies

$$
\hat{\boldsymbol{F}} \cdot(-\boldsymbol{n})>0 .
$$

(P3) The two bodies have a tendency to separate after the collision event

$$
\Delta \boldsymbol{v}_{2} \cdot(-n) \geq 0
$$

(P4) The dissipation of kinetic energy during the collision event is non-negative

$$
T_{1}-T_{2} \geq 0
$$

\section{A constitutive equation for the impulsive force}

Once a constitutive equation for the impulsive force $\hat{\boldsymbol{F}}$ is specified the motion of the two particles just after collision is determined by the equations (2-3). This section presents a constitutive equation for $\hat{\boldsymbol{F}}$. First it is noted that with the help of (2-9) and (2-12), the restrictions (2-14) and (2-15) require

$$
-\boldsymbol{f} \cdot \Delta \boldsymbol{v}_{1}>0, \quad-\boldsymbol{f} \cdot \boldsymbol{n}>0 .
$$

Also, using (2-13), the restriction (2-17) on energy dissipation requires

$$
\begin{aligned}
& 1+\eta>0, \\
& 1-\eta \geq 0 .
\end{aligned}
$$

The work in [Maw et al. 1976; 1981] and [Walton 1993, Sec. 25.5.1] suggests that a model for hardparticle collision between two spheres can be formulated in terms of $\psi_{1}$ in (2-8) and $\psi_{2}$ defined by

$$
\psi_{1}=\frac{\Delta \boldsymbol{v}_{1} \cdot \boldsymbol{\tau}}{\Delta \boldsymbol{v}_{1} \cdot \boldsymbol{n}} \geq 0, \quad \psi_{2}=-\left[\frac{\Delta \boldsymbol{v}_{2} \cdot \boldsymbol{\tau}}{\Delta \boldsymbol{v}_{2} \cdot \boldsymbol{n}}\right]
$$


The parameters $\left\{\psi_{1}, \psi_{2}\right\}$ are measures of the obliquity of the collision event, just before and just after the collision, respectively. Moreover, the model in [Walton 1993] uses the standard coefficient of restitution $e$, which connects the normal components of relative velocities by

$$
\Delta \boldsymbol{v}_{2} \cdot \boldsymbol{n}=-e\left(\Delta \boldsymbol{v}_{1} \cdot \boldsymbol{n}\right), \quad 0 \leq e \leq 1
$$

which with the help of (2-9) ensures that the restriction (2-16) is satisfied. Here, use is made of (3-4) to obtain a constitutive equation for $\eta$. Specifically, from (2-6) and (2-12) it can be shown that (3-4) yields the constitutive equation

$$
(1+\eta)=(1+e)\left[\frac{\boldsymbol{A} \cdot(\boldsymbol{f} \otimes \boldsymbol{f})}{\boldsymbol{A} \cdot(-\boldsymbol{f} \otimes \boldsymbol{n})}\right]\left(\frac{\boldsymbol{n} \cdot \Delta \boldsymbol{v}_{1}}{-\boldsymbol{f} \cdot \Delta \boldsymbol{v}_{1}}\right) .
$$

The hard-particle model proposed by Walton [1993, Sec. 25.5.1] introduced a rotational coefficient of restitution $\beta$ associated with the tangential direction $\tau$ defined by

$$
\Delta \boldsymbol{v}_{2} \cdot \boldsymbol{\tau}=-\beta\left(\Delta \boldsymbol{v}_{1} \cdot \boldsymbol{\tau}\right), \quad-1 \leq \beta \leq 1
$$

Here, this constitutive assumption is replaced by a constitutive equation for a shear coefficient $\mu$, which is the ratio of the shearing component of the impulsive force to its normal component. Specifically, it is assumed that the impulsive force is in the $\boldsymbol{n}-\boldsymbol{\tau}$ plane with

$$
\boldsymbol{f}=-\frac{1}{\sqrt{1+\mu^{2}}}(\boldsymbol{n}+\mu \boldsymbol{\tau}), \quad \mu=\tan \phi>0,
$$

where $\phi$ is the friction angle (see Figure 1). Then, using (2-8) it can be shown that the restrictions (3-1) are automatically satisfied

$$
-\boldsymbol{f} \cdot \Delta \boldsymbol{v}_{1}=\frac{\left|\Delta \boldsymbol{v}_{1}\right|}{\sqrt{1+\psi_{1}^{2}} \sqrt{1+\mu^{2}}}\left(1+\mu \psi_{1}\right)>0, \quad-\boldsymbol{f} \cdot \boldsymbol{n}=\frac{1}{\sqrt{1+\mu^{2}}}>0 .
$$

It should be noted that although the impulsive force has been assumed to limited to the $\boldsymbol{n}-\boldsymbol{\tau}$ plane this does not mean that the motion of the spheres is two-dimensional since the angular velocities of the spheres need not be normal to this collision plane. Next, using (2-6), (2-8) and (3-7) it can be shown that

$$
1+\eta=\left(\frac{1+e}{1+\mu \psi_{1}}\right)\left[1+\left(\frac{1+I_{m}}{I_{m}}\right) \mu^{2}\right]>0,
$$

which shows that the restriction (3-2a) is automatically satisfied. Then, the restriction (3-2b) can be rewritten in the form

$$
1-\eta=2-\left(\frac{1}{1+\mu \psi_{1}}\right)\left[1+\left(\frac{1+I_{m}}{I_{m}}\right) \mu^{2}\right] \geq 0,
$$

which imposes a non-trivial restriction on the functional form for the shear coefficient $\mu$.

In order to motivate a functional form for $\mu$ use is made of (2-6), (2-8), (2-12), (3-3) (3-7), (3-8) and (3-9) to deduce that 


$$
\begin{aligned}
\hat{\boldsymbol{F}} & =-m(1+e) \frac{\left|\Delta \boldsymbol{v}_{1}\right|}{\sqrt{1+\psi_{1}^{2}}}(\boldsymbol{n}+\mu \boldsymbol{\tau}), \\
\Delta \boldsymbol{v}_{2} & =\frac{e\left|\Delta \boldsymbol{v}_{1}\right|}{\sqrt{1+\psi_{1}^{2}}}\left(-\boldsymbol{n}+\psi_{2} \boldsymbol{\tau}\right) \\
\psi_{2} & =\frac{1}{e}\left[\psi_{1}-(1+e)\left(\frac{1+I_{m}}{I_{m}}\right) \mu\right] .
\end{aligned}
$$

The hard-particle model in [Walton 1993] assumes that $\psi_{2}$ is a bi-linear function of $\psi_{1}$ with a negative slope in the region of partial slip and a positive slope in the region of full slip. Motivated by this model, a constitutive equation for $\mu$ is specified by the form

$$
\mu=\mu_{s} g(z), \quad g(z)=1-\langle 1-z\rangle^{1+\alpha}, \quad \alpha>0, \quad z=\frac{\psi_{1}}{\psi_{s}}, \quad \psi_{s}=(1+e)\left(\frac{1+I_{m}}{I_{m}}\right) \mu_{s},
$$

where $\langle x\rangle=\max (x, 0)$ denotes the Macaulay brackets. Thus, $\psi_{2}$ in (3-11) can be simplified to obtain

$$
\psi_{2}=\psi_{2}\left(\psi_{1}\right)=\frac{\psi_{s}}{e}[z-g(z)]
$$

It follows that

$$
\psi_{2}(0)=0, \quad \frac{d \psi_{2}}{d \psi_{1}}(0)=-\frac{\alpha}{e}, \quad \psi_{2}\left(\psi_{s}\right)=0, \quad \frac{d \psi_{2}}{d \psi_{1}}\left(\psi_{s}\right)=\frac{1}{e} .
$$

This means that the measure $\psi_{2}$ of the obliquity angle just after the collision is a smooth function of the measure $\psi_{1}$ of the obliquity angle just before the collision. The response characterizes partial slip with negative values of $\psi_{2}$ for $\psi_{1}<\psi_{s}$ and full slip with positive values of $\psi_{2}$ for $\psi_{1}>\psi_{s}$. Moreover, the transition to full slip occurs when $\psi_{2}$ changes sign with $\psi_{1}=\psi_{s}$. Also, the shear coefficient $\mu$ attains and maintains its maximum constant value $\mu_{s}$ during full slip.

This hard-particle model is characterized by three model parameters

$$
\left\{e, \mu_{s}, \alpha\right\}
$$

with $\alpha$ controlling the shape of the transition region from partial slip to full slip. The model will satisfy the physical restrictions (2-14)-(2-17) on the impulsive force when the values of these parameters ensure that (3-10) is satisfied for all collision events. In the following sections this model is compared with the hard-particle model of Walton [1993] and examples are considered which demonstrate the importance of the smooth transition in the partial slip region.

\section{The hard-particle collision model of Walton [1993]}

The hard-particle model proposed by Walton [1993, Sec. 25.5.1] is characterized by the usual coefficient of restitution $e$ in (3-4) and a rotational coefficient of restitution $\beta$ associated with the tangential direction $\boldsymbol{\tau}$ defined in (3-6) (see Figure 2). These expressions tacitly assume that $\Delta \boldsymbol{v}_{2}$ remains in the plane of $\left\{n, \Delta \boldsymbol{v}_{1}\right\}$ with

$$
\Delta \boldsymbol{v}_{2}=-\left[e\left(\Delta \boldsymbol{v}_{1} \cdot \boldsymbol{n}\right) \boldsymbol{n}+\beta\left(\Delta \boldsymbol{v}_{1} \cdot \boldsymbol{\tau}\right) \boldsymbol{\tau}\right],
$$


which with the help of (2-8) and (3-3) can be rewritten in the form

$$
\Delta \boldsymbol{v}_{2}=\frac{e\left|\Delta \boldsymbol{v}_{1}\right|}{\sqrt{1+\psi_{1}^{2}}}\left(-\boldsymbol{n}+\psi_{2} \boldsymbol{\tau}\right), \quad \psi_{2}=-\frac{\beta}{e} \psi_{1} .
$$

Moreover, Walton [1993] proposed a functional form for $\beta$ defined by

$$
\beta=\min \left(\beta_{0}, \beta^{*}\right), \quad \beta^{*}=-1+\frac{\psi_{s}}{\psi_{1}}, \quad 0<\beta_{0} \leq 1,
$$

where $\psi_{s}$ is given by (3-12) and it is noted that $K$ in [Walton 1993] is equal to $I_{m}$ here. It then follows that (4-2) and (4-3) yield

$$
\psi_{2}=-\frac{\beta_{0}}{e} \psi_{1} \text { for } 0 \leq \psi_{1} \leq \frac{\psi_{s}}{1+\beta_{0}}, \quad \psi_{2}=\frac{1}{e}\left(\psi_{1}-\psi_{s}\right) \text { for } \psi_{1} \geq \frac{\psi_{s}}{1+\beta_{0}},
$$

which satisfy the conditions (3-14) when $\beta_{0}=\alpha$. Moreover, using (3-12) the expression (3-11) can be rewritten in the form

$$
\psi_{2}=\frac{1}{e}\left[\psi_{1}-\psi_{s}\left(\frac{\mu}{\mu_{s}}\right)\right]
$$

Thus, comparing (4-2) and (4-5), replacing $\left\{\mu, \beta_{0}\right\}$ by $\left\{\mu_{W}, \alpha\right\}$ and using the definition of $z$ in (3-12) yields the constitutive equation

$$
\mu=\mu_{W}=(1+\beta) \mu_{s} z, \quad \beta=\min \left(\alpha, \beta^{*}\right), \quad \beta^{*}=-1+\frac{\psi_{s}}{\psi_{1}},
$$

which causes the model described in Sections 2 and 3 to be the same as Walton's hard-particle model. The associated value $\eta_{W}$ of $\eta$ is given by (3-9) with $\mu$ replaced by $\mu_{W}$

$$
\eta=\eta_{W}=1-\left(\frac{1}{1+\mu_{W} \psi_{1}}\right)\left[1+\left(\frac{1+I_{m}}{I_{m}}\right) \mu_{W}^{2}\right] .
$$

This functional form automatically satisfies the restriction

$$
1+\eta_{W}>0
$$

and must satisfy the restriction

$$
1-\eta_{W} \geq 0
$$

in order to ensure non-negative energy dissipation during the collision.

In summary, Walton's hard-particle model is given by (4-5)-(4-9) which depend on the same three parameters (3-15) as the smooth transition model proposed in Section 3.

\section{Comparison of the smooth model with Walton's model}

In the remainder of the paper attention is limited to the case of uniform spheres for which

$$
I_{A}=\frac{2}{5} m_{A} R_{A}^{2}, \quad I_{B}=\frac{2}{5} m_{B} R_{B}^{2}, \quad I_{m}=\frac{2}{5} .
$$




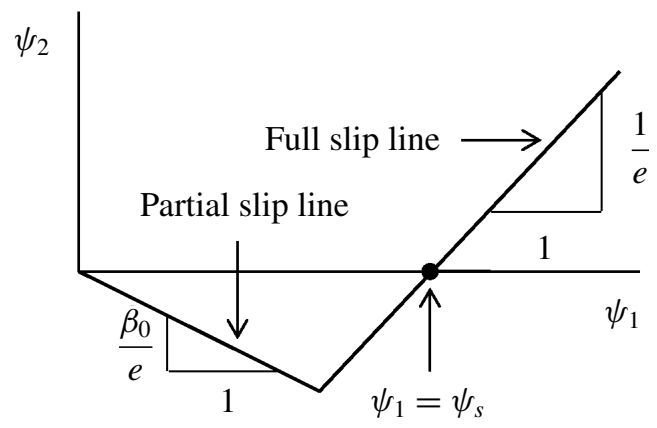

Figure 2. Sketch of the hard-sphere collision model of [Walton 1993].

The results in Figures 3-5 compare the response of the smooth model (denoted by $S$ ) with that of Walton's model (denoted by $W$ ) for

$$
\mu_{s}=0.3, \quad e=1 \text { or } 0.8, \quad \alpha=0.35,1 \text { or } 5 .
$$

where the value of $\alpha=0.35$ was suggested in [Walton 1993]. In these figures the subscript has been removed from $\mu_{W}$. These figures show that the differences between the two models can be significant, especially for the larger values of $\alpha$ when $e=0.8$. In particular, it can be seen from Figure 3(c) that for the smooth model the transition to full slip occurs at an obliquity angle $\theta=64.5^{\circ}$ so the transition region from no slip to full slip is large. Also, it can be seen from parts (d) and (e) of Figure 4 that during this transition region Walton's model predicts no dissipation whereas the smooth model predicts dissipation
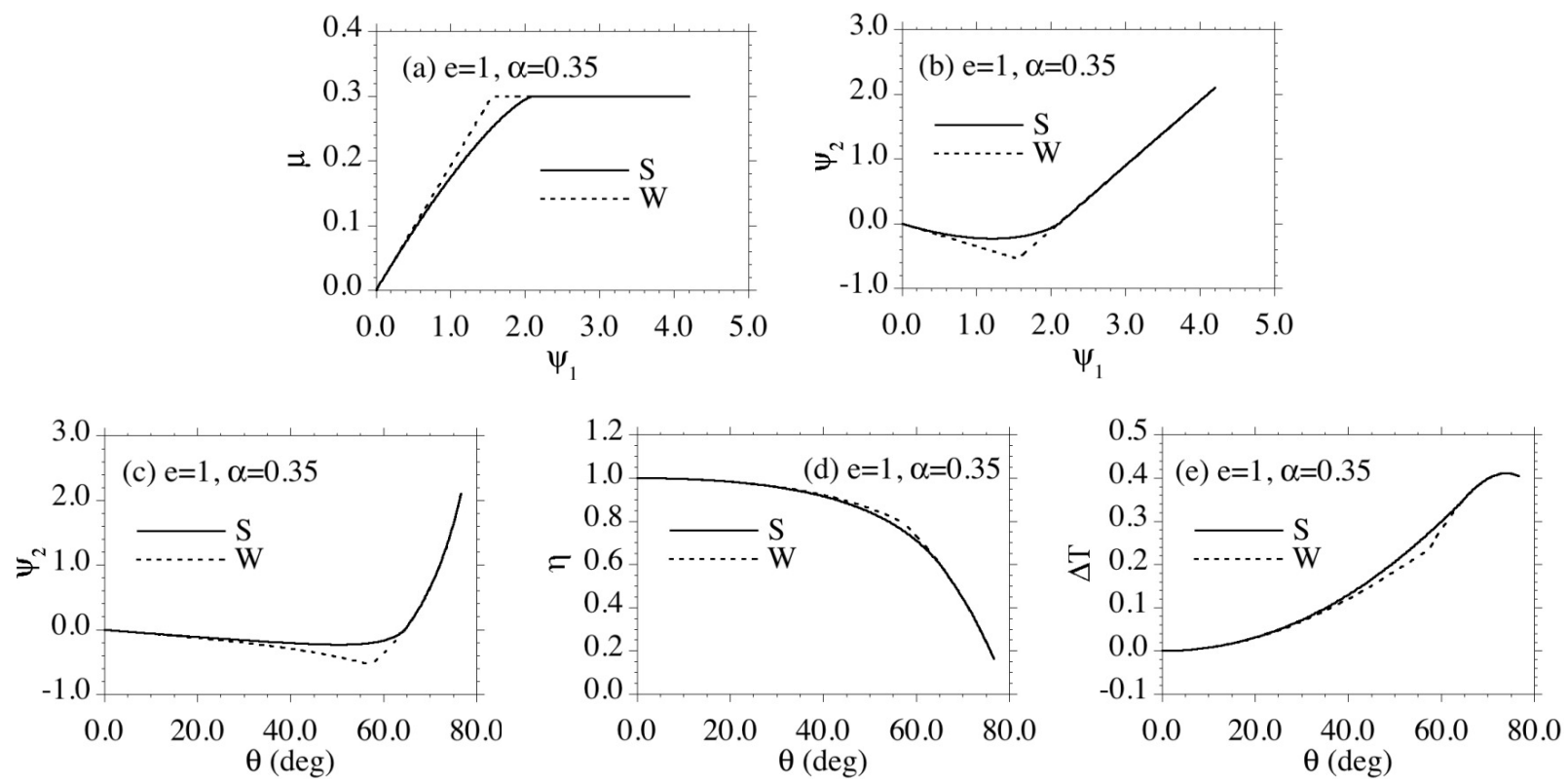

Figure 3. Comparison of the smooth model $(S)$ with Walton's model $(W)$ for $e=1$, $\alpha=0.35$ and $\mu_{s}=0.3$. 

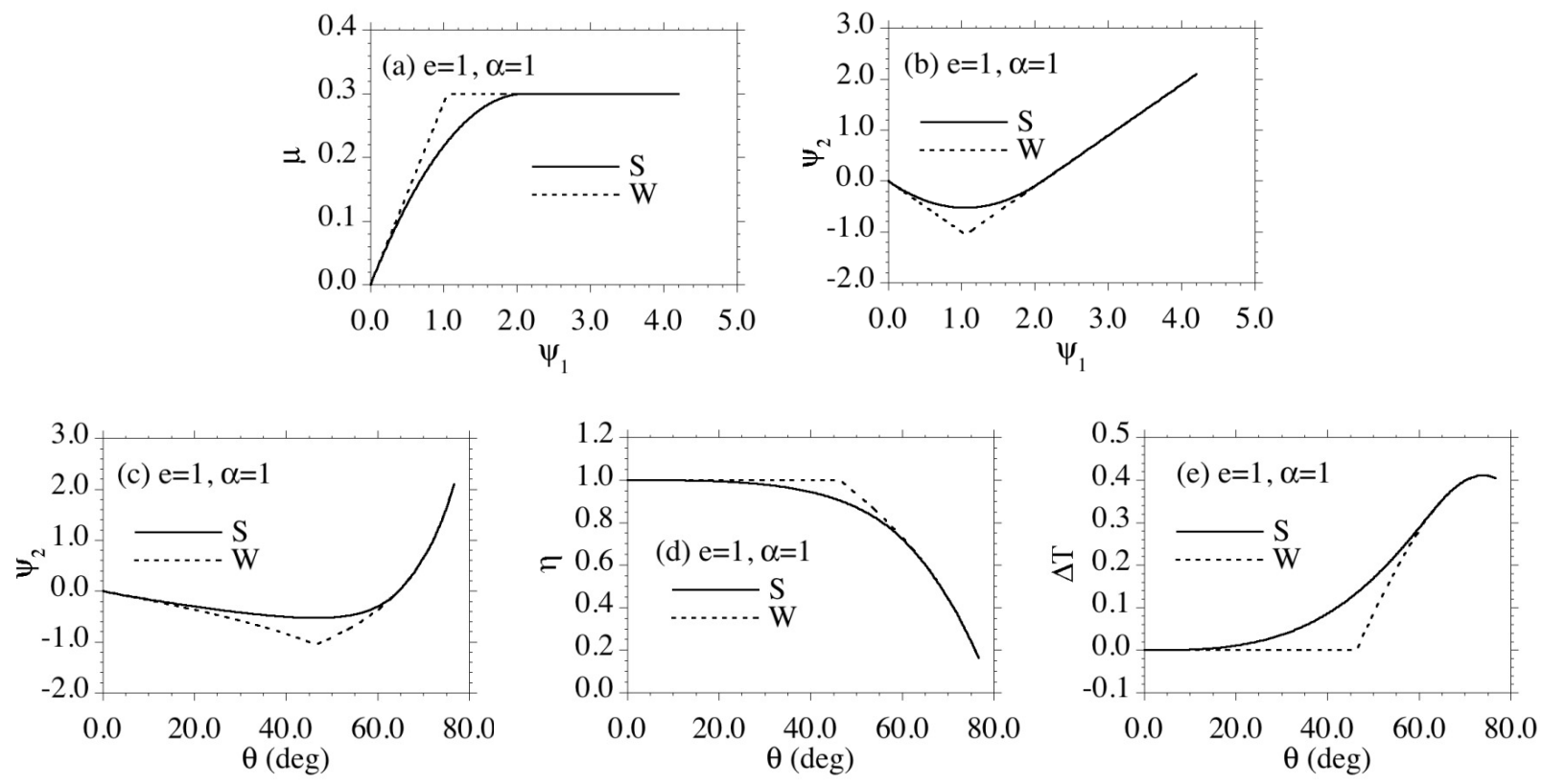

Figure 4. Comparison of the smooth model $(S)$ with Walton's model $(W)$ for $e=1$, $\alpha=1$ and $\mu_{s}=0.3$.
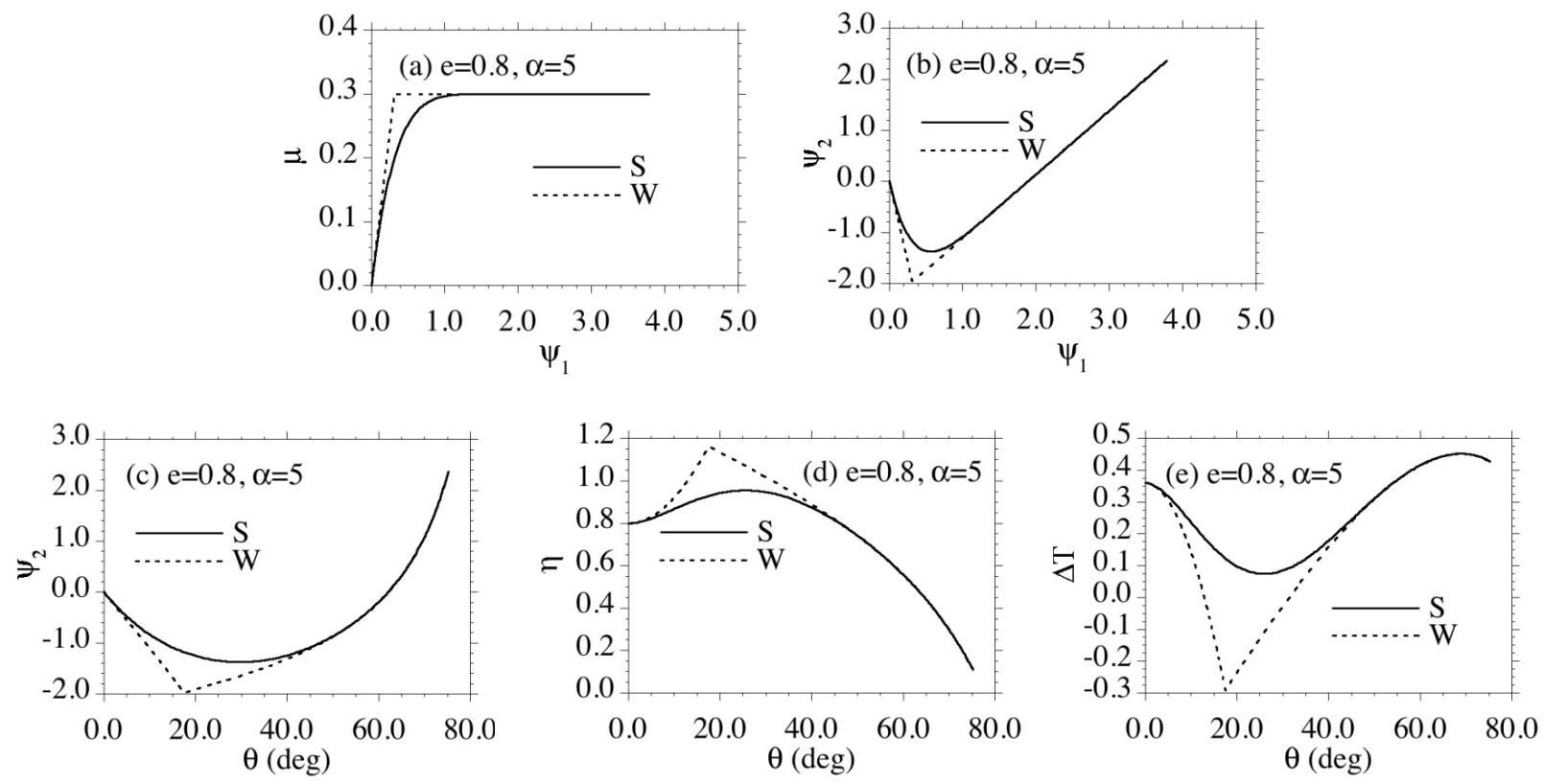

Figure 5. Comparison of the smooth model $(S)$ with Walton's model $(W)$ for $e=0.8$, $\alpha=5$ and $\mu_{s}=0.3$. 
over the entire range of the response. The results in Figures 3 and 4 satisfy the dissipation inequalities (3-9) and (3-10). Moreover, the parameters used for Figure 5 show that these inequalities are not trivially satisfied. In particular, it can be seen from parts (d) and (e) of Figure 5 that Walton's model would violate the dissipation inequality (3-10), while the smooth model would satisfy the dissipation inequality (3-10).

\section{Bouncing of a sphere off a fixed horizontal plane}

Figure 6 shows a sketch of a uniform sphere $(A)$ colliding with a fixed horizontal plane with attention limited to the vertical $\boldsymbol{e}_{1}-\boldsymbol{e}_{2}$ plane. Also, the motion of each sphere is simplified to be two-dimensional with its angular velocity in the $\boldsymbol{e}_{3}$ direction. The body force of gravity $b$ per unit mass acts in the positive $\boldsymbol{e}_{1}$ direction. The effect of gravity is used to determine the motion of the sphere between collision events but is neglected during the collisions. This approximation causes the model to limited to strong collision events.

Using the formulation in the previous sections the wall can be modeled by the sphere $(B)$ with the specifications

$$
m_{B} \gg m_{A}, \quad R_{B} \gg R_{A}, \quad \boldsymbol{v}_{B 1}=\boldsymbol{v}_{B 2}=\boldsymbol{v}_{D 1}=\boldsymbol{v}_{D 2}=0, \quad \boldsymbol{\omega}_{B 1}=\omega_{B 2}=0 .
$$

Next, consider a general collision event which occurs at $t=t_{n}$ with

$$
\begin{aligned}
\boldsymbol{v}_{A 1}\left(t_{n}\right) & =u_{1}\left(t_{n}\right) \boldsymbol{e}_{1}+w_{1}\left(t_{n}\right) \boldsymbol{e}_{2}, \quad u_{1}\left(t_{n}\right)>0, \quad \boldsymbol{\omega}_{A 1}\left(t_{n}\right)=\omega_{1}\left(t_{n}\right) \boldsymbol{e}_{3}, \\
\boldsymbol{v}_{A 2}\left(t_{n}\right) & =u_{2}\left(t_{n}\right) \boldsymbol{e}_{1}+w_{2}\left(t_{n}\right) \boldsymbol{e}_{2}, \quad u_{2}\left(t_{n}\right) \leq 0, \quad \boldsymbol{\omega}_{A 2}\left(t_{n}\right)=\omega_{2}\left(t_{n}\right) \boldsymbol{e}_{3}, \\
\Delta \boldsymbol{v}_{1}\left(t_{n}\right) & =\boldsymbol{v}_{C 1}\left(t_{n}\right)=u_{1}\left(t_{n}\right) \boldsymbol{e}_{1}+\left[w_{1}\left(t_{n}\right)+R_{A} \omega_{1}\left(t_{n}\right)\right] \boldsymbol{e}_{2}, \\
\Delta \boldsymbol{v}_{2}\left(t_{n}\right) & =\boldsymbol{v}_{C 2}\left(t_{n}\right)=u_{2}\left(t_{n}\right) \boldsymbol{e}_{1}+\left[w_{2}\left(t_{n}\right)+R_{A} \omega_{2}\left(t_{n}\right)\right] \boldsymbol{e}_{2}, \\
\boldsymbol{n} & =\boldsymbol{e}_{1}, \quad \boldsymbol{\tau}\left(t_{n}\right)=\frac{s_{1}\left(t_{n}\right)}{\left|s_{1}\left(t_{n}\right)\right|} \boldsymbol{e}_{2}, \quad s_{1}\left(t_{n}\right)=w_{1}\left(t_{n}\right)+R_{A} \omega_{1}\left(t_{n}\right),
\end{aligned}
$$
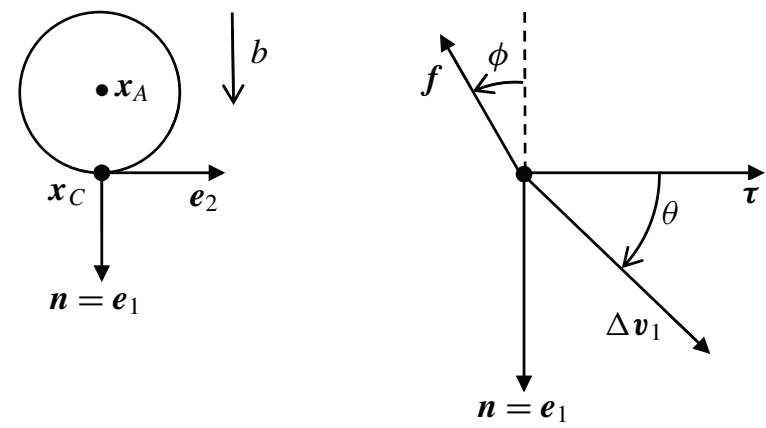

Figure 6. Sketch of a sphere colliding with a fixed horizontal plane showing the normal $\boldsymbol{n}$ and tangent $\boldsymbol{\tau}$ unit vectors and the directions of $\Delta \boldsymbol{v}_{1}$ and $\boldsymbol{f}$. 
where $s_{1}\left(t_{n}\right)$ is the slip velocity just before collision. Then, with the help of (2-3), (2-6)-(2-8), (2-13), (3-3), (3-7)-(3-9), (3-11)-(3-13), (5-1), (6-1) and (6-2) it follows that

$$
\begin{aligned}
& m=m_{A}, \quad \psi_{s}=\frac{7}{2}(1+e) \mu_{s}, \quad \eta_{n}=-1+\left[\frac{1+e+\mu_{s} \psi_{s} g_{n}^{2}}{1+\mu_{s} \psi_{s} z_{n} g_{n}}\right], \\
& \psi_{1}\left(t_{n}\right)=\frac{\left|s_{1}\left(t_{n}\right)\right|}{u_{1}\left(t_{n}\right)}, \quad z_{n}=\frac{\psi_{1}\left(t_{n}\right)}{\psi_{s}}, \quad g_{n}=1-\left\langle 1-z_{n}\right\rangle^{\alpha+1}, \\
& \psi_{2}\left(t_{n}\right)=-\frac{s_{1}\left(t_{n}\right)\left[w_{2}\left(t_{n}\right)+R_{A} \omega_{2}\left(t_{n}\right)\right]}{\left|s_{1}\left(t_{n}\right)\right| u_{2}\left(t_{n}\right)}, \quad \hat{\boldsymbol{F}}_{n}=-m_{A}(1+e) u_{1}\left(t_{n}\right)\left[\boldsymbol{e}_{1}+\mu_{s} g_{n} \frac{s_{1}\left(t_{n}\right)}{\left|s_{1}\left(t_{n}\right)\right|} \boldsymbol{e}_{2}\right], \\
& u_{2}\left(t_{n}\right)=-e u_{1}\left(t_{n}\right), \quad w_{2}\left(t_{n}\right)=w_{1}\left(t_{n}\right)-\frac{2}{7} u_{1}\left(t_{n}\right) \psi_{s} g_{n}\left[\frac{s_{1}\left(t_{n}\right)}{\left|s_{1}\left(t_{n}\right)\right|}\right], \\
& R_{A} \omega_{2}\left(t_{n}\right)=R_{A} \omega_{1}\left(t_{n}\right)-\frac{5}{7} u_{1}\left(t_{n}\right) \psi_{s} g_{n}\left[\frac{s_{1}\left(t_{n}\right)}{\left|s_{1}\left(t_{n}\right)\right|}\right] .
\end{aligned}
$$

Also, with the help of (5-1) the kinetic energy $T\left(t_{n}\right)$ just before the collision is given by

$$
T\left(t_{n}\right)=\frac{1}{2} m_{A}\left[u_{1}^{2}\left(t_{n}\right)+w_{1}^{2}\left(t_{n}\right)+\frac{2}{5} R_{A}^{2} \omega_{1}^{2}\left(t_{n}\right)\right] .
$$

Next, expressing the center of mass $\boldsymbol{x}_{A}$ and the angular velocity $\boldsymbol{\omega}_{A}$ of the sphere in the forms

$$
\boldsymbol{x}_{A}=x \boldsymbol{e}_{1}+y \boldsymbol{e}_{2}, \quad \boldsymbol{\omega}_{A}=\omega \boldsymbol{e}_{3},
$$

the balance laws of linear and angular momentum of the sphere between collisions require

$$
\ddot{x}=b, \quad \ddot{y}=0, \quad \dot{\omega}=0 .
$$

Using the values $\left\{u_{1}\left(t_{n}\right), w_{1}\left(t_{n}\right), \omega_{1}\left(t_{n}\right)\right\}$ just before the collision at $t=t_{n}$, the equations (6-3) determine the initial conditions $\left\{u_{2}\left(t_{n}\right), w_{2}\left(t_{n}\right), \omega_{2}\left(t_{n}\right)\right\}$ just after collision, with the initial values of $\{x, y, \omega\}$ for (6-6) given by

$$
x\left(t_{n}\right)=0, \quad \dot{x}\left(t_{n}\right)=u_{2}\left(t_{n}\right), \quad y\left(t_{n}\right)=y\left(t_{n}^{-}\right), \quad \dot{y}\left(t_{n}\right)=w_{2}\left(t_{n}\right), \quad \omega\left(t_{n}\right)=\omega_{2}\left(t_{n}\right),
$$

where $y\left(t_{n}^{-}\right)$is the value of $y$ just before the collision. Then, the solution of (6-6) is given by

$$
\begin{aligned}
& x(t)=u_{2}\left(t_{n}\right)\left(t-t_{n}\right)+\frac{1}{2} b\left(t-t_{n}\right)^{2}, \\
& y(t)=y\left(t_{n}\right)+w_{2}\left(t_{n}\right)\left(t-t_{n}\right), \\
& \omega(t)=\omega_{2}\left(t_{n}\right) \text { for } t_{n} \leq t \leq t_{n+1} .
\end{aligned}
$$

The value $t=t_{n+1}$ is the time when the next collision occurs with the wall

$$
x\left(t_{n+1}\right)=0 \Rightarrow t_{n+1}=t_{n}-\frac{2 u_{2}\left(t_{n}\right)}{b},
$$

with the value $y\left(t_{n+1}\right)$ for the collision at $t=t_{n+1}$ being specified by

$$
y\left(t_{n+1}\right)=y\left(t_{n}\right)-\frac{2 u_{2}\left(t_{n}\right) w_{2}\left(t_{n}\right)}{b} .
$$



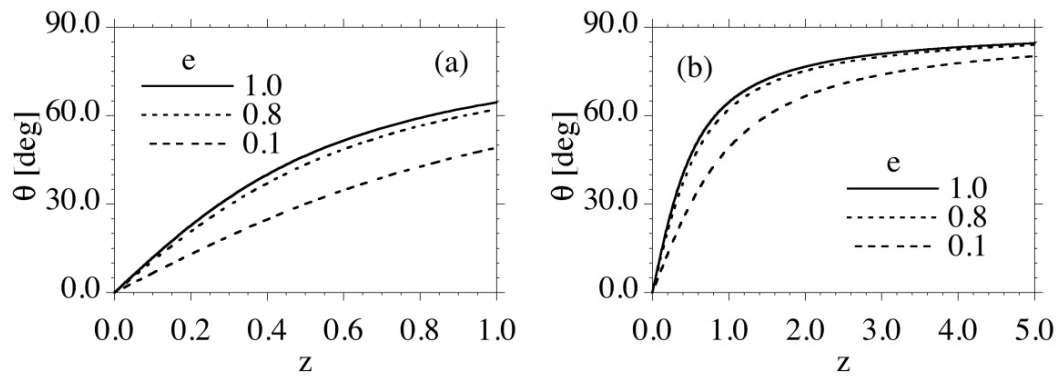

Figure 7. Values of the obliquity angle $\theta$ as a function of the normalized variable $z$.
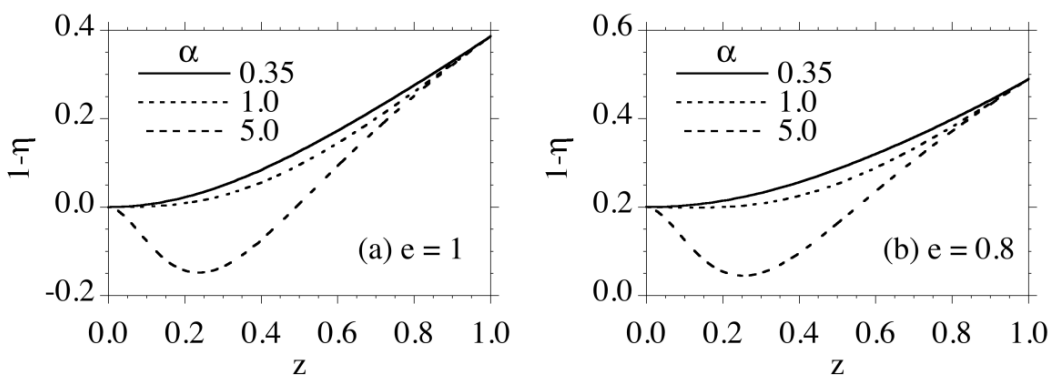

Figure 8. Examination of the restriction $1-\eta>0$ for positive energy dissipation on the parameters $e$ and $\alpha$.

Moreover, the values of $\left\{u_{1}\left(t_{n+1}\right), w_{1}\left(t_{n+1}\right), \omega_{1}\left(t_{n+1}\right)\right\}$ just before the collision at $t=t_{n+1}$ are given by

$$
u_{1}\left(t_{n+1}\right)=-u_{2}\left(t_{n}\right), \quad w_{1}\left(t_{n+1}\right)=w_{2}\left(t_{n}\right), \quad \omega_{1}\left(t_{n+1}\right)=\omega_{2}\left(t_{n}\right),
$$

which with the help of (6-3) are used to determine the initial conditions $\left\{u_{2}\left(t_{n+1}\right), w_{2}\left(t_{n+1}\right), \omega_{2}\left(t_{n+1}\right)\right\}$ just after collision at $t=t_{n+1}$. Then, the process is repeated for additional collisions.

Before presenting the solution to bouncing, it is of interest to examine the dependence of the incident obliquity angle $\theta$ and the quantity $(1-\eta)$, which characterizes energy dissipation, on the parameter $z$ for different values of the model parameters. For the examples in this section $\mu_{s}$ is specified by the value in (5-2) and the solution is considered for different values of $e$ and $\alpha$. Specifically, use is made of (2-8), (3-10), (3-12), and (6-3) to determine values for $\theta$ and (1- 1 ). Figure 7 plots $\theta$ as a function of $z$ for different values of $e$. From this figure it can be seen that the obliquity angles $\theta$ for $e=1$ and 0.8 are greater than $60^{\circ}$ at the transition to full slip $(z=1)$ so the transition region with partial slip is significant. Figure 8 plots $(1-\eta)$ as a function of $z$ for different values of $e$ and $\alpha$. Recalling that $(1-\eta)$ must be positive for positive energy dissipation during the collision, it can be seen that for $e=1$, the value of $\alpha$ has to be less than or equal to unity for positive energy dissipation, but for lower values of $e$ the value of $\alpha$ can be greater than unity and still satisfy the restriction of positive energy dissipation. 
In order to interpret the results of specific examples, it is convenient to define the following normalized parameters

$$
\begin{array}{lll}
U_{n}=\frac{u_{1}\left(t_{n}\right)}{u_{1}\left(t_{1}\right)}, & W_{n}=\frac{w_{1}\left(t_{n}\right)}{u_{1}\left(t_{1}\right)}, & \Omega_{n}=\frac{R_{A} \omega_{1}\left(t_{n}\right)}{u_{1}\left(t_{1}\right)}, \\
S_{n}=\frac{s_{1}\left(t_{n}\right)}{\psi_{s} u_{1}\left(t_{1}\right)}=\frac{W_{n}+\Omega_{n}}{\psi_{s}}, & \bar{T}_{n}=\frac{T\left(t_{n}\right)}{\frac{1}{2} m_{A} u_{1}^{2}\left(t_{n}\right)}=U_{n}^{2}+W_{n}^{2}+\frac{2}{5} \Omega_{n}^{2}, & E_{n}=\frac{\bar{T}_{n}}{\bar{T}_{1}},
\end{array}
$$

where $\left\{U_{n}, W_{n}, \Omega_{n}, S_{n}, \bar{T}_{n}, E_{n}\right\}$ characterize, respectively, the vertical velocity, horizontal velocity, angular velocity, slip velocity, kinetic energy and the energy reduction just before the collision at $t=t_{n}$. Then, equations (6-3), (6-11) and (6-12) yield

$$
\begin{aligned}
& z_{n}=\frac{\left|S_{n}\right|}{U_{n}}, \quad g_{n}=1-\left\langle 1-z_{n}\right\rangle^{1+\alpha}, \quad \eta_{n}=-1+\left[\frac{1+e+\mu_{s} \psi_{s} g_{n}^{2}}{1+\mu_{s} \psi_{s} z_{n} g_{n}}\right], \\
& U_{n+1}=e U_{n}, \quad W_{n+1}=W_{n}-\frac{2}{7} \psi_{s}\left(\frac{S_{n}}{z_{n}}\right) g_{n}, \quad \Omega_{n+1}=\Omega_{n}-\frac{5}{7} \psi_{s}\left(\frac{S_{n}}{z_{n}}\right) g_{n}, \\
& S_{n+1}=S_{n} G_{n}, \quad G_{n}\left(z_{n}\right)=1-\frac{g_{n}}{z_{n}} .
\end{aligned}
$$

Next, consider special state defined by

$$
W_{n}=\frac{1}{7}(2+5 \gamma) \psi_{s} S_{n}, \quad \Omega_{n}=\frac{5}{7}(1-\gamma) \psi_{s} S_{n}, \quad \bar{T}_{n}=U_{n}^{2}+\frac{1}{7} \psi_{s}^{2} S_{n}^{2}\left(2+5 \gamma^{2}\right) .
$$

From these expressions it can be seen that the two states $\gamma= \pm|\gamma|$, with different values of the horizontal velocity $W_{n}$ and angular velocity $\Omega_{n}$, have the same slip velocity $S_{n}$ and the same kinetic energy $\bar{T}_{n}$. Moreover, it follows from (6-13) and (6-14) that

$$
\begin{aligned}
W_{n+1} & =\frac{1}{7}(2+5 \bar{\gamma}) \psi_{s} S_{n+1}, & \Omega_{n+1} & =\frac{5}{7}(1-\bar{\gamma}) \psi_{s} S_{n+1}, \\
\bar{T}_{n+1} & =U_{n+1}^{2}+\frac{1}{7} \psi_{s}^{2} S_{n+1}^{2}\left(2+5 \bar{\gamma}^{2}\right), & \bar{\gamma} & =\frac{\gamma}{1-\frac{g_{n}}{z_{n}}} .
\end{aligned}
$$

Comparison of (6-14) and (6-15) indicates that consecutive collisions maintain the same structure so the response of the slip velocity $S_{n}$ and the energy reduction $E_{n}$ for the two initial states specified by

$$
U_{1}=1, \quad W_{1}=\frac{1}{7}(2+5 \gamma) \psi_{s} S_{1}, \quad \Omega_{1}=\frac{5}{7}(1-\gamma) \psi_{s} S_{1}, \quad \bar{T}_{1}=1+\frac{1}{7} \psi_{s}^{2} S_{1}^{2}\left(2+5 \gamma^{2}\right),
$$

with $\gamma= \pm|\gamma|$ are the same. This means that the parameters in the model can be determined by experiments or simulations for which the sphere has no initial angular velocity $\{\gamma=1\}$ and the results can be used for general states. In the following examples the initial state is specified by (6-16) with

$$
S_{1}=z_{1}>0
$$

From (6-13) it follows that when $e$ is less than unity, the value of the vertical velocity $U_{n}$ decreases with each collision, eventually attaining the value of zero. The function $G_{n}\left(z_{n}\right)$ in (6-13) which characterizes the slip velocity satisfies the properties

$$
G_{n}(0)=-\alpha, \quad G_{n}(1)=0, \quad G_{n}(\infty)=1, \quad \frac{d G_{n}}{d z_{n}} \geq 0, \quad-\alpha \leq G\left(z_{n}\right) \leq 1 .
$$


Moreover, it can be shown that

$$
-1<G_{n}\left(z_{n}\right)<1 \text { for } 0<\alpha<1 \text { and } 0 \leq z_{n}<\infty
$$

which in view of (6-13) means that the slip velocity decreases during each collision as long as $z_{n}$ remains bounded. However, the rate of decrease in $U_{n}$ might be slower than that of $S_{n}$, causing $z_{n}$ to approach infinity and the value of $S_{n}$ to approach a non-zero constant.

6.1. Bouncing vertically with no slip. The simplest special solution is one where the sphere does not translate horizontally and does not rotate with

$$
z_{n}=0, \quad W_{n}=0, \quad \Omega_{n}=0, \quad S_{n}=0 \text { for } n=1,2, \ldots
$$

This solution is valid for any values of $\left\{e, \alpha, \mu_{s}\right\}$ and consists of the sphere merely bouncing vertically. If $e$ is less than unity, the sphere loses energy during each collision and eventually stops bouncing.

6.2. Bouncing vertically with initial slip. For this example the initial slip velocity has the special value

$$
S_{1}=z_{1}=1, \quad W_{1}+\Omega_{1}=\psi_{s},
$$

which allows the sphere to initially have nonzero horizontal velocity $W_{1}$ and nonzero angular velocity $\Omega_{1}$. Since $G_{n}(1)=0$ in (6-13) it follows that slip velocity $S_{n}$ reduces to zero during the first collision and remains zero

$$
S_{n}=0 \text { for } z_{1}=1 \text { and } n=2,3, \ldots
$$

This result is also independent of the values of $\{e, \alpha\}$. Although the slip velocity might be zero this does not mean that the sphere has stopped rotating or translating. In particular, it can be seen from (6-14)-(6-15) that $\left\{W_{n}, \Omega_{n}\right\}$ will both vanish when $S_{n}$ vanishes if

$$
\gamma=0, \quad \frac{W_{n}}{\Omega_{n}}=\frac{2}{5} \text { for } n=1,2, \ldots
$$

6.3. Bouncing with partial energy dissipation. For an example of bouncing with partial energy dissipation, the parameters are specified by (6-16) and (6-17) with

$$
e=1, \quad \alpha=0.35, \quad \mu_{s}=0.3, \quad \gamma=1, \quad z_{1}=0.5
$$

Figure 9 shows the response of this sphere which is initially not rotating. Since $e=1$, the vertical velocity $U_{n}$ before each collision shown in part (a) of the figure remains constant so there is only partial energy dissipation. The slip velocity $S_{n}$ in part (b) reduces to zero, the horizontal velocity $W_{n}$ in part (c) attains a constant positive value and the energy reduction $E_{n}$ in part (d) decreases to a constant positive value. The sphere continues to bounce, translating and rotating without slip at the contact points of the collisions.

6.4. Bouncing with dissipation of the vertical kinetic energy. For an example of bouncing with dissipation of the vertical kinetic energy, the parameters are specified by

$$
e=0.8, \quad \alpha=0.35, \quad \mu_{s}=0.3, \quad \gamma=0, \quad z_{1}=1
$$



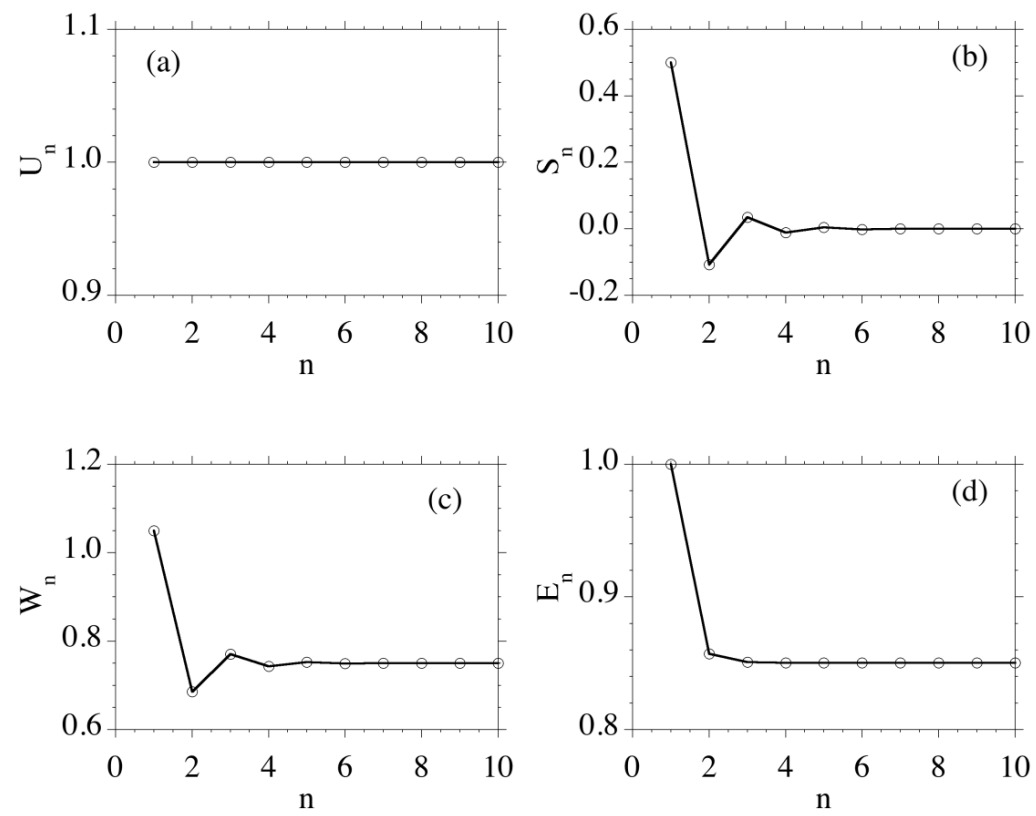

Figure 9. Bouncing with partial energy dissipation: The solution shows initial slip with $\left\{S_{n}, W_{n}\right\}$. The parameters are specified by $e=1, \alpha=0.35, \mu_{s}=0.3, \gamma=1, z_{1}=0.5$.

Figure 10 shows that for these special values $\left\{\gamma=0, z_{1}=1\right\}$ the nonzero initial values of the slip velocity $S_{n}$, the horizontal velocity $W_{n}$ and the angular velocity $\Omega_{n}$ all drop to zero during the first collision and remain zero. This means that after the first collision the sphere bounces vertically until all its kinetic energy is dissipated.

6.5. Bouncing with dissipation of the vertical kinetic energy and continued slip. For an example of bouncing with dissipation of the vertical kinetic energy and continued slip the parameters are specified by

$$
e=0.8, \quad \alpha=0.35, \quad \mu_{s}=0.3, \quad \gamma=1, \quad z_{1}=6
$$

which has a much larger value of the slip velocity and obliquity angle than that in (6-25). Figure 11(a) shows that the normal velocity $U_{n}$ decays to zero; in the rest of the figure it can be seen that the slip velocity $S_{n}$ (b), the horizontal velocity $W_{n}$ (c) and the energy reduction $E_{n}(\mathrm{~d})$ all reduce to positive values. In particular, the normal velocity $U_{n}$ decays faster than the slip velocity $S_{n}$, causing $z_{n}$ to approach infinity with $G_{n}$ in (6-13) approaching zero. Also, it is interesting to note that when $z_{n}$ approaches infinity the parameter $\eta$ approaches $(-1)$.

As $U_{n}$ approaches zero the sphere chatters (bouncing with imperceptible amplitude). The kinetic energy remains constant even though the sphere continues to slip because the force of gravity is neglected during these small collision events. This is an unphysical result of the approximation that the impulsive force dominates the bounded force of gravity during the collision and it identifies a limitation of the model for certain situations with multiple collision events. 

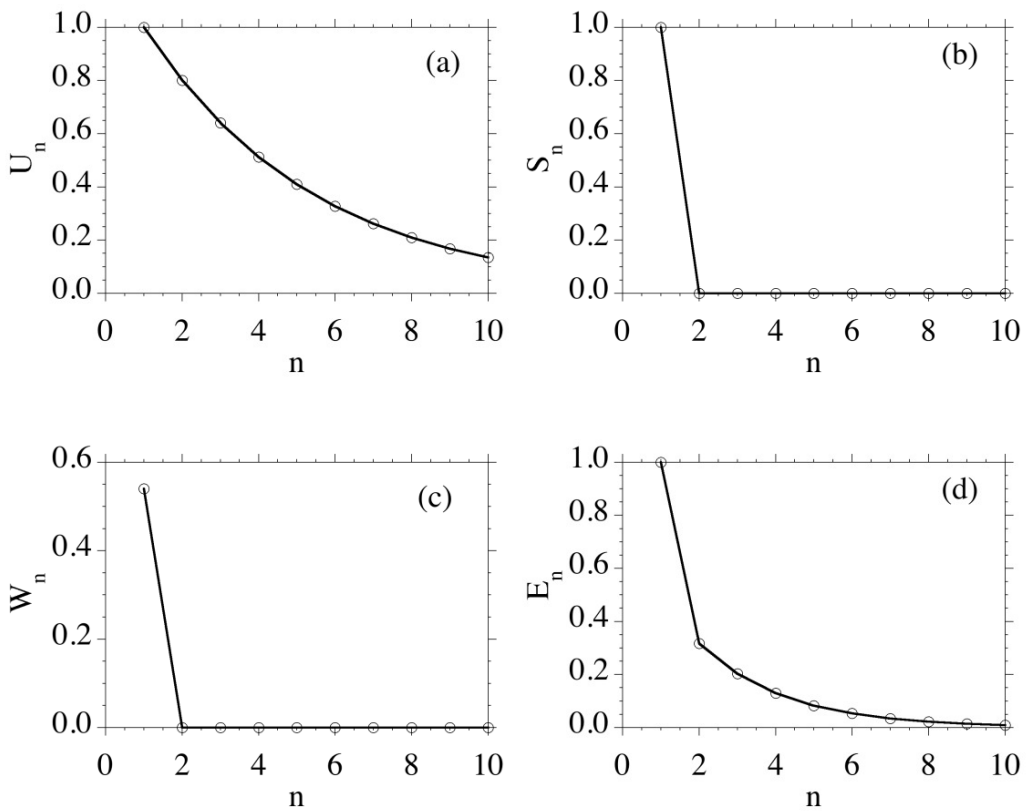

Figure 10. Bouncing with dissipation of the vertical kinetic energy: The solution shows initial slip with $\left\{S_{n}, W_{n}, \Omega_{n}\right\}$ dropping to zero after the first collision. The parameters are specified by $e=0.8, \alpha=0.35, \mu_{s}=0.3, \gamma=0, z_{1}=1$.
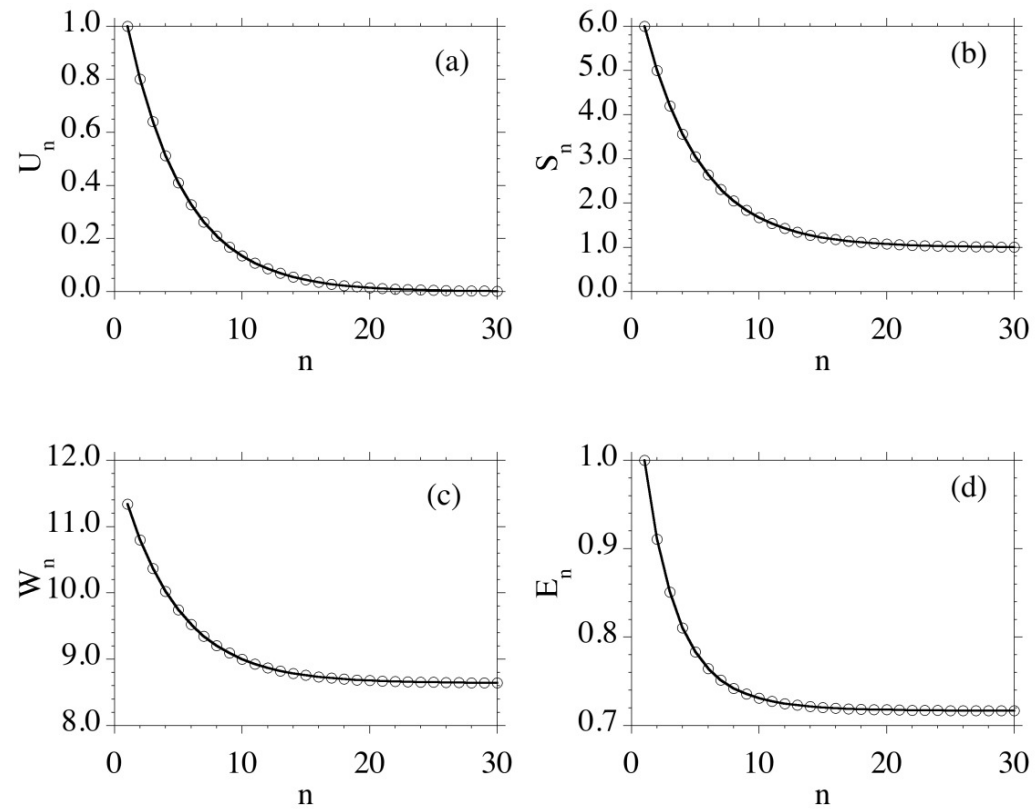

Figure 11. Bouncing with dissipation of the vertical kinetic energy: The solution shows continued slipping $\left(S_{n}>0\right)$ with the parameters specified by $e=0.8, \alpha=0.35, \mu_{s}=0.3$, $\gamma=1, z_{1}=6$. 


\section{Conclusions}

The smooth transition model for hard-particle collision of spheres presented here has been motivated by the model of Walton [1993], which has a sharp transition between a region of partial slip for low obliquity angles and a region of full slip for higher obliquity angles. Specifically, the notion of a coefficient of rotational restitution in the Walton model is replaced by a constitutive equation for the shear coefficient $\mu$, which controls the ratio of the shear component of the impulsive force to its normal component. The model depends on three parameters: the standard coefficient of restitution $e$ for relative normal velocities; a constant value $\mu_{s}$ of friction for sliding in the full slip region; and a parameter $\alpha$ in the function $g(z)$ in (3-12) that controls the shape of the transition region from partial to full slip.

The resulting smooth transition model is relatively simple and the three model parameters $\left\{e, \mu_{s}, \alpha\right\}$ can be evaluated from experimental data or from simulations using soft-particle contact models. Examples show that the smooth transition region is large with the obliquity angle $\theta$ being between 0 and nearly $60^{\circ}$. Moreover, it is shown that the physical condition of non-negative energy dissipation imposes nontrivial restrictions on the model parameters.

\section{Acknowledgements}

The author would like to acknowledge helpful discussions with $\mathrm{O}$ Walton. Also, this research was partially supported by MB Rubin's Gerard Swope Chair in Mechanics.

\section{References}

[Chatterjee and Ruina 1998] A. Chatterjee and A. Ruina, "A new algebraic rigid-body collision law based on impulse space considerations”, J. Appl. Mech. (ASME) 65:4 (1998), 939-951.

[Deen et al. 2007] N. G. Deen, M. Van Sint Annaland, M. A. Van der Hoef, and J. A. M. Kuipers, "Review of discrete particle modeling of fluidized beds", Chem. Eng. Sci. 62:1-2 (2007), 28-44.

[Di Renzo and Di Maio 2004] A. Di Renzo and F. P. Di Maio, "Comparison of contact-force models for the simulation of collisions in DEM-based granular flow codes”, Chem. Eng. Sci. 59:3 (2004), 525-541.

[Goldsmith 1960] W. Goldsmith, Impact: the theory and physical behaviour of colliding solids, Edward Arnold, London, 1960.

[Ivanov 1992] A. P. Ivanov, “Энергетика удара с трением”, Prikl. Mat. Mekh. 56:4 (1992), 624-631. Translated as "Energetics of a collision with friction" in J. Appl. Math. Mech. 56:4 (1992), 527-534.

[Ketterhagen et al. 2005] W. R. Ketterhagen, J. S. Curtis, and C. R. Wassgren, "Stress results from two-dimensional granular shear flow simulations using various collision models", Phys. Rev. E 71:6 (2005), Article ID \#061307.

[Kosinski and Hoffmann 2010] P. Kosinski and A. C. Hoffmann, "An extension of the hard-sphere particle-particle collision model to study agglomeration”, Chem. Eng. Sci. 65:10 (2010), 3231-3239.

[Kosinski and Hoffmann 2011] P. Kosinski and A. C. Hoffmann, "Extended hard-sphere model and collisions of cohesive particles", Phys. Rev. E 84:3 (2011), Article ID \#031303. Erratum in 89:4 (2014), Article ID \#049901.

[Kruggel-Emden et al. 2007] H. Kruggel-Emden, E. Simsek, S. Rickelt, S. Wirtz, and V. Scherer, "Review and extension of normal force models for the discrete element method", Powder Technol. 171:3 (2007), 157-173.

[Liu et al. 2011] G. Liu, S. Li, and Q. Yao, "A JKR-based dynamic model for the impact of micro-particle with a flat surface", Powder Technol. 207:1-3 (2011), 215-223.

[Lu et al. 2005] H. Lu, S. Wang, Y. Zhao, L. Yang, D. Gidaspow, and J. Ding, "Prediction of particle motion in a twodimensional bubbling fluidized bed using discrete hard-sphere model”, Chem. Eng. Sci. 60:12 (2005), 3217-3231.

[Maw et al. 1976] N. Maw, J. R. Barber, and J. N. Fawcett, “The oblique impact of elastic spheres", Wear 38:1 (1976), $101-114$. 
[Maw et al. 1981] N. Maw, J. R. Barber, and J. N. Fawcett, "The role of elastic tangential compliance in oblique impact", J. Lubr. Technol. (ASME) 103:1 (1981), 74-80.

[Meyer and Deglon 2011] C. J. Meyer and D. A. Deglon, "Particle collision modeling: a review", Miner. Eng. 24:8 (2011), 719-730.

[Morris et al. 2004] J. P. Morris, M. B. Rubin, S. C. Blair, L. A. Glenn, and F. E. Heuze, "Simulations of underground structures subjected to dynamic loading using the distinct element method", Eng. Comput. (Swansea Wales) 21:2-4 (2004), 384-408.

[Prasanth et al. 2012] P. S. Prasanth, J. K. Kakkassery, and R. Vijayakumar, "A variable hard sphere-based phenomenological inelastic collision model for rarefied gas flow simulations by the direct simulation Monte Carlo method", Fluid Dyn. Res. 44:2 (2012), Article ID \#025503.

[Rubin 1998] M. B. Rubin, "Physical restrictions on the impulse acting during three-dimensional impact of two 'rigid' bodies", J. Appl. Mech. (ASME) 65:2 (1998), 464-469.

[Stevens and Hrenya 2005] A. B. Stevens and C. M. Hrenya, "Comparison of soft-sphere models to measurements of collision properties during normal impacts", Powder Technol. 154:2-3 (2005), 99-109.

[Walton 1993] O. R. Walton, "Numerical simulation of inelastic frictional particle-particle interactions", Chapter 25, pp. 884911 in Particulate two-phase flow, edited by M. C. Roco, Butterworth-Heinemann, Boston, 1993.

[Walton 2004] O. R. Walton, "Potential discrete element simulation applications ranging from airborne fines to pellet beds", SAE Technical Paper 2004-01-2329, 2004.

[Zhu et al. 2007] H. P. Zhu, Z. Y. Zhou, R. Y. Yang, and A. B. Yu, "Discrete particle simulation of particulate systems: theoretical developments", Chem. Eng. Sci. 62:13 (2007), 3378-3396.

Received 23 May 2015. Accepted 20 Sep 2015.

M. B. RUBIN: mbrubin@tx.technion.ac.il

Faculty of Mechanical Engineering, Technion - Israel Institute of Technology, 32000 Haifa, Israel 


\title{
JOURNAL OF MECHANICS OF MATERIALS AND STRUCTURES
}

\author{
msp.org/jomms
}

Founded by Charles R. Steele and Marie-Louise Steele

\section{EDITORIAL BOARD}

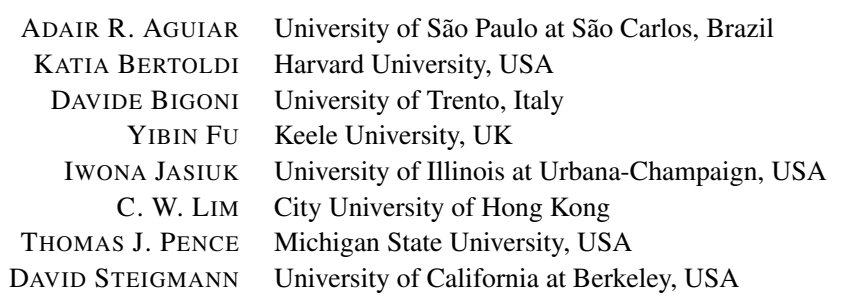

\section{ADVISORY BOARD}

J. P. CARTER University of Sydney, Australia

D. H. Hodges Georgia Institute of Technology, USA

J. HUTCHINSON Harvard University, USA

D. PAMPlona Universidade Católica do Rio de Janeiro, Brazil

M. B. RUBin Technion, Haifa, Israel

PRODUCTION production@msp.org

SILvio Levy Scientific Editor

See msp.org/jomms for submission guidelines.

JoMMS (ISSN 1559-3959) at Mathematical Sciences Publishers, 798 Evans Hall \#6840, c/o University of California, Berkeley, CA 94720-3840, is published in 10 issues a year. The subscription price for 2016 is US \$575/year for the electronic version, and $\$ 735 /$ year ( $\$ 60$, if shipping outside the US) for print and electronic. Subscriptions, requests for back issues, and changes of address should be sent to MSP.

JoMMS peer-review and production is managed by EditFLOW ${ }^{\circledR}$ from Mathematical Sciences Publishers.

PUBLISHED BY

mathematical sciences publishers

nonprofit scientific publishing

http://msp.org/

(C) 2016 Mathematical Sciences Publishers 


\title{
Journal of Mechanics of Materials and Structures
}

\author{
Volume 11, No. $2 \quad$ March 2016
}

The effect of small scale on the free vibration of functionally graded truncated conical shells

Yaghoub TAdi Beni and Fahimeh Mehralian

Conditions for the localisation of plastic deformation in temperature sensitive viscoplastic materials MARTIN K. PAESOLd, ANDREW P. BASSOM, KLAUS RegenaUeR-Lieb and Manolis VeVEAKIS

A simple hard-particle collision model with a smooth transition to full slip

Multiobjective optimization of laminated composite plate with elliptical cut-out using ANN based NSGA-II

P. Emmanuel Nicholas, M. C. Lenin Babu and A. Sathya Sofia

Analytical estimates for the lateral thrust in bolted steel buckling-restrained braces Guido Bregoli, Francesco Genna and Giovanni Metelli 